MATHEMATICS OF COMPUTATION

Volume 73, Number 247, Pages 1139-1152

S 0025-5718(03)01600-4

Article electronically published on August 19, 2003

\title{
ANALYSIS OF RECOVERY TYPE A POSTERIORI ERROR ESTIMATORS FOR MILDLY STRUCTURED GRIDS
}

\author{
JINCHAO XU AND ZHIMIN ZHANG
}

\begin{abstract}
Some recovery type error estimators for linear finite elements are analyzed under $O\left(h^{1+\alpha}\right)(\alpha>0)$ regular grids. Superconvergence of order $O\left(h^{1+\rho}\right)(0<\rho \leq \alpha)$ is established for recovered gradients by three different methods. As a consequence, a posteriori error estimators based on those recovery methods are asymptotically exact.
\end{abstract}

\section{INTRODUCTION}

A posteriori error estimates have become standard in modern engineering and scientific computation. There are two types of popular error estimators: the residual type (see, e.g., [2], 4]) and the recovery type (see, e.g., [21]). The most representative recovery type error estimator is the Zienkiewicz-Zhu error estimator, especially the estimator based on gradient patch recovery by local discrete least-squares fitting 22], [23. The method is now widely used in engineering practice for its robustness in a posteriori error estimates and its efficiency in computer implementation. It is a common belief that the robustness of the $\mathrm{ZZ}$ estimator is rooted in the superconvergence property of the associated gradient recovery under structured meshes. Superconvergence properties of the ZZ recovery based on local least-squares fitting are proven by Zhang [17] for all popular elements under rectangular meshes, by Li-Zhang [1] for linear elements under strongly regular triangular meshes, and by Zhang-Victory [18] for tensor product elements under strongly regular quadrilateral meshes.

While there is a sizable literature on theoretical investments for residual type error estimators (see, e.g., [1], [3], [10], [14] and references therein), there have not been many theoretical results on recovery type error estimators. Nevertheless, the recovery type error estimators perform astonishingly well even for unstructured grids. The current paper intends to explain this phenomenon. We observe that for

Received by the editor June 26, 2002 and, in revised form, December 15, 2002.

2000 Mathematics Subject Classification. Primary 65N30; Secondary 65N50, 65N15, 65N12, 65D10, 74S05, 41A10, 41A25.

Key words and phrases. Gradient recovery, ZZ patch recovery, superconvergence, a posteriori error estimates.

The work of the first author was supported in part by the National Science Foundation grant DMS-9706949 and the Center for Computational Mathematics and Applications, Penn State University.

The work of the second author was supported in part by the National Science Foundation grants DMS-0074301, DMS-0079743, and INT-0196139. 
an unstructured mesh, when adaptive procedure is used, a mesh refinement will usually bring in some kind of local structure. It is then reasonable to assume that for most of the domain, every two adjacent triangles form an $O\left(h^{1+\alpha}\right)$ approximate parallelogram. Under this assumption, we are able to establish superconvergence of the gradient recovery operator for three popular methods: weighted averaging, local $L^{2}$-projection, and the $\mathrm{ZZ}$ patch recovery. Furthermore, by utilizing an integral identity for linear elements on one triangular element developed by Bank and Xu [5], we are able to generalize their superconvergence result between the finite element solution and the linear interpolation from an $O\left(h^{2}\right)$ regular grid to an $O\left(h^{1+\alpha}\right)$ regular grid. Finally, we are able to prove asymptotic exactness of the three recovery error estimators.

The topic of a posteriori error estimates has recently attracted more and more attention in the scientific community (see, e.g., [5], [6], [7], [9], [16], 20]; also see recent books [1], 3] for some general discussions). The literature regarding finite element superconvergence theory can be found in the books [8], 10, 12], [15], [19].

\section{Geometry identities of A tRIANGLE}

In this section, we shall generalize the result in [5] for $\alpha=1$ to all $\alpha>0$. Following the argument in [5], we consider in Figure 1] a triangle $\tau$ with vertices $\boldsymbol{p}_{k}^{t}=\left(x_{k}, y_{k}\right), 1 \leq k \leq 3$, oriented counterclockwise, and corresponding nodal basis functions (barycentric coordinates) $\left\{\phi_{k}\right\}_{k=1}^{3}$. Let $\left\{e_{k}\right\}_{k=1}^{3}$ denote the edges of element $\tau,\left\{\theta_{k}\right\}_{k=1}^{3}$ the angles, $\left\{\boldsymbol{n}_{k}\right\}_{k=1}^{3}$ the unit outward normal vectors, $\left\{\boldsymbol{t}_{k}\right\}_{k=1}^{3}$ the unit tangent vectors with counterclockwise orientation, $\left\{\ell_{k}\right\}_{k=1}^{3}$ the edge lengths, and $\left\{d_{k}\right\}_{k=1}^{3}$ the perpendicular heights. Let $\tilde{\boldsymbol{p}}$ be the point of intersection for the perpendicular bisectors of the three sides of $\tau$. Let $\left|s_{k}\right|$ denote the distance between $\tilde{\boldsymbol{p}}$ and side $k$. If $\tau$ has no obtuse angles, then the $s_{k}$ will be nonnegative. Otherwise, the distance to the side opposite the obtuse angle will be negative.

Let $\mathcal{D}_{\tau}$ be a symmetric $2 \times 2$ matrix with constant entries. We define

$$
\xi_{k}=-\boldsymbol{n}_{k+1} \cdot \mathcal{D}_{\tau} \boldsymbol{n}_{k-1} .
$$

The important special case $\mathcal{D}_{\tau}=I$ corresponds to $-\Delta$, and in this case $\xi_{k}=\cos \theta_{k}$. Let $q_{k}=\phi_{k+1} \phi_{k-1}$ denote the quadratic bump function associated with edge $e_{k}$ and let $\psi_{k}=\phi_{k}\left(1-\phi_{k}\right)$.

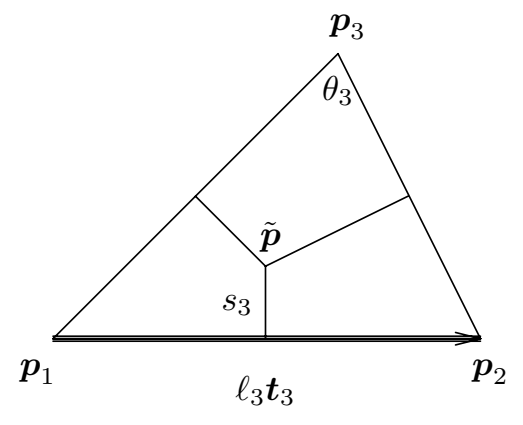

Figure 1. Parameters associated with the triangle $\tau$

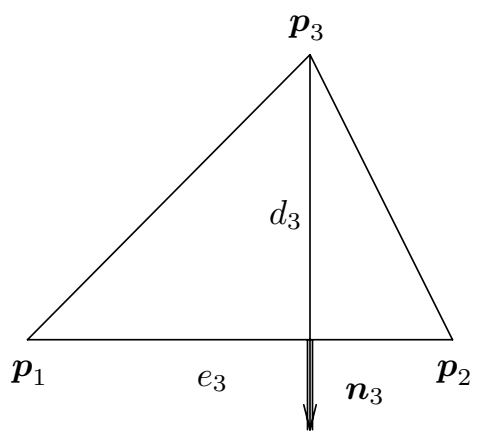


The following fundamental identity is proved in [5] for $v_{h} \in P_{1}(\tau)$ :

$$
\begin{gathered}
\int_{\tau} \nabla\left(u-u_{I}\right) \cdot \mathcal{D}_{\tau} \nabla v_{h}=\sum_{k=1}^{3} \int_{e_{k}} \frac{\xi_{k} q_{k}}{2 \sin \theta_{k}}\left\{\left(\ell_{k+1}^{2}-\ell_{k-1}^{2}\right) \frac{\partial^{2} u}{\partial \boldsymbol{t}_{k}^{2}}+4|\tau| \frac{\partial^{2} u}{\partial \boldsymbol{t}_{k} \partial \boldsymbol{n}_{k}}\right\} \frac{\partial v_{h}}{\partial \boldsymbol{t}_{k}} \\
-\int_{\tau} \sum_{k=1}^{3} \frac{\ell_{k} \xi_{k}}{2 \sin ^{2} \theta_{k}}\left\{\ell_{k+1} \psi_{k-1} \frac{\partial^{3} u}{\partial^{2} \boldsymbol{t}_{k+1} \partial \boldsymbol{t}_{k-1}}+\ell_{k-1} \psi_{k+1} \frac{\partial^{3} u}{\partial^{2} \boldsymbol{t}_{k-1} \partial \boldsymbol{t}_{k+1}}\right\} \frac{\partial v_{h}}{\partial \boldsymbol{t}_{k}},
\end{gathered}
$$

where $u_{I} \in P_{1}(\tau)$ is the linear interpolation of $u$ on $\tau$.

We say that two adjacent triangles (sharing a common edge) form an $O\left(h^{1+\alpha}\right)$ $(\alpha>0)$ approximate parallelogram if the lengths of any two opposite edges differ only by $O\left(h^{1+\alpha}\right)$.

Definition. The triangulation $\mathcal{T}_{h}=\mathcal{T}_{1, h} \cup \mathcal{T}_{2, h}$ is said to satisfy Condition $(\alpha, \sigma)$ if there exist positive constants $\alpha$ and $\sigma$ such that every two adjacent triangles inside $\mathcal{T}_{1, h}$ form an $O\left(h^{1+\alpha}\right)$ parallelogram and

$$
\bar{\Omega}_{1, h} \cup \bar{\Omega}_{2, h}=\bar{\Omega}, \quad\left|\Omega_{2, h}\right|=O\left(h^{\sigma}\right), \quad \bar{\Omega}_{i, h} \equiv \bigcup_{\tau \in \mathcal{T}_{i, h}} \bar{\tau}, \quad i=1,2 .
$$

Remark. There are two important ingredients in an automatic mesh generation code. One, called swap diagonal, changes the direction of some diagonal edges in order to obtain near parallel directions for adjacent element edges and to make as many nodes as possible have six triangles attached. Another, known as Lagrange smoothing, iteratively relocates nodes to place each node near a mesh symmetry center (see condition (3.1) in Section 3).

Clearly, both swap diagonal and Lagrange smoothing are intended to make every two adjacent triangles form an $O\left(h^{1+\alpha}\right)$ parallelogram. Eventually, only a small portion of elements (including boundary elements) do not satisfy this condition. These elements then belong to $\Omega_{2, h}$, which has a small measure. Therefore, Condition $(\alpha, \sigma)$ is a reasonable condition in practice and can be satisfied by most meshes produced by automatic mesh generation codes.

Denote $\mathcal{V}_{h} \subset H^{1}(\Omega)$, the $C^{0}$ linear finite element space associated with $\mathcal{T}_{h}$.

Lemma 2.1. Assume that $\mathcal{T}_{h}$ satisfy Condition $(\alpha, \sigma)$. Let $\mathcal{D}_{\tau}$ be a piecewise constant matrix function defined on $\mathcal{T}_{h}$, whose elements $\mathcal{D}_{\text {rij }}$ satisfy

$$
\left|\mathcal{D}_{\tau i j}\right| \lesssim 1, \quad\left|\mathcal{D}_{\tau i j}-\mathcal{D}_{\tau^{\prime} i j}\right| \lesssim h^{\alpha}, \quad i=1,2 ; j=1,2 .
$$

Here $\tau$ and $\tau^{\prime}$ are a pair of triangles sharing a common edge. Then for any $v_{h} \in \mathcal{V}_{h}$ (2.2)

$$
\left|\sum_{\tau \in \mathcal{T}_{h}} \int_{\tau} \nabla\left(u-u_{I}\right) \cdot \mathcal{D}_{\tau} \nabla v_{h}\right| \lesssim h^{1+\rho}\left(\|u\|_{3, \Omega}+|u|_{2, \infty, \Omega}\right)|v|_{1, \Omega}, \quad \rho=\min \left(\alpha, \frac{\sigma}{2}, \frac{1}{2}\right),
$$

where $u_{I} \in \mathcal{V}_{h}$ is the interpolation of $u$.

Proof. Applying (2.1),

$$
\sum_{\tau \in \mathcal{T}_{h}} \int_{\tau} \nabla\left(u-u_{I}\right) \cdot \mathcal{D}_{\tau} \nabla v_{h}=I_{1}+I_{2}
$$


where

$$
\begin{aligned}
& I_{1}= \sum_{\tau \in \mathcal{T}_{h}} \sum_{k=1}^{3} \int_{e_{k}} \frac{\xi_{k} q_{k}}{2 \sin \theta_{k}}\left\{\left(\ell_{k+1}^{2}-\ell_{k-1}^{2}\right) \frac{\partial^{2} u}{\partial \boldsymbol{t}_{k}^{2}}+4|\tau| \frac{\partial^{2} u}{\partial \boldsymbol{t}_{k} \partial \boldsymbol{n}_{k}}\right\} \frac{\partial v_{h}}{\partial \boldsymbol{t}_{k}}, \\
& I_{2}=-\sum_{\tau \in \mathcal{T}_{h}} \int_{\tau} \sum_{k=1}^{3} \frac{\ell_{k} \xi_{k}}{2 \sin ^{2} \theta_{k}} \\
& \quad \times\left\{\ell_{k+1} \psi_{k-1} \frac{\partial^{3} u}{\partial^{2} \boldsymbol{t}_{k+1} \partial \boldsymbol{t}_{k-1}}+\ell_{k-1} \psi_{k+1} \frac{\partial^{3} u}{\partial^{2} \boldsymbol{t}_{k-1} \partial \boldsymbol{t}_{k+1}}\right\} \frac{\partial v_{h}}{\partial \boldsymbol{t}_{k}} .
\end{aligned}
$$

$I_{2}$ is easily estimated by

$$
\left|I_{2}\right| \lesssim h^{2}\|u\|_{3, \Omega}\left|v_{h}\right|_{1, \Omega}
$$

To estimate $I_{1}$, we separate all interior edges into two different groups. $\mathcal{E}_{1}$ is the set of edges $e$ such that the two adjacent triangles sharing $e$ form an $O\left(h^{1+\alpha}\right)$ approximate parallelogram and $\mathcal{E}_{2}$ is the set of the remaining interior edges. The set of all interior edges is given by $\mathcal{E}=\mathcal{E}_{1}+\mathcal{E}_{2}$.

For each $e \in \mathcal{E}$, there are two triangles, say $\tau$ and $\tau^{\prime}$, that share $e$ as a common edge. Denote, with respect to $\tau$,

$$
\alpha_{e}=\frac{\xi_{k}}{2 \sin \theta_{k}}\left(\ell_{k+1}^{2}-\ell_{k-1}^{2}\right), \quad \beta_{e}=\frac{\xi_{k}}{2 \sin \theta_{k}} 4|\tau|,
$$

and with respect to $\tau^{\prime}$,

$$
\alpha_{e}^{\prime}=\frac{\xi_{k^{\prime}}}{2 \sin \theta_{k^{\prime}}}\left(\ell_{k^{\prime}+1}^{2}-\ell_{k^{\prime}-1}^{2}\right), \quad \beta_{e}^{\prime}=\frac{\xi_{k^{\prime}}}{2 \sin \theta_{k^{\prime}}} 4\left|\tau^{\prime}\right| .
$$

Taking $\boldsymbol{n}$ and $\boldsymbol{t}$ to correspond to $\tau$, we can write

$$
I_{1}=I_{11}+I_{12}+I_{13},
$$

where

$$
I_{1 j}=\sum_{e \in \mathcal{E}_{j}} \int_{e} q_{e}\left\{\left(\alpha_{e}-\alpha_{e}^{\prime}\right) \frac{\partial^{2} u}{\partial \boldsymbol{t}^{2}}+\left(\beta_{e}-\beta_{e}^{\prime}\right) \frac{\partial^{2} u}{\partial \boldsymbol{t} \partial \boldsymbol{n}}\right\} \frac{\partial v_{h}}{\partial \boldsymbol{t}}
$$

for $j=1,2$, and

$$
I_{13}=\sum_{e \subset \partial \Omega} \int_{e} q_{e}\left\{\alpha_{e} \frac{\partial^{2} u}{\partial \boldsymbol{t}^{2}}+\beta_{e} \frac{\partial^{2} u}{\partial \boldsymbol{t} \partial \boldsymbol{n}}\right\} \frac{\partial v_{h}}{\partial \boldsymbol{t}} .
$$

It is easy to see that, if $v_{h}=0$ on $\partial \Omega$, then $I_{13}=0$. Otherwise, we have the following estimate:

$$
\left|I_{13}\right| \lesssim h^{3 / 2}|u|_{2, \infty, \partial \Omega}\left|v_{h}\right|_{1, \Omega}
$$

Setting $\boldsymbol{z}=\boldsymbol{t}$ and $\boldsymbol{z}=\boldsymbol{n}$, we estimate

$$
\left|\int_{e} q_{e} \frac{\partial^{2} u}{\partial \boldsymbol{t} \partial \boldsymbol{z}} \frac{\partial v_{h}}{\partial \boldsymbol{t}}\right| \lesssim h^{-1}|u|_{2, \infty, \Omega} \int_{\tau}\left|\nabla v_{h}\right| .
$$

By definition, for $e \in \mathcal{E}_{1}, \alpha_{e}^{\prime}=\alpha_{e}\left(1+O\left(h^{\alpha}\right)\right)$ and $\beta_{e}^{\prime}=\beta_{e}\left(1+O\left(h^{\alpha}\right)\right)$. Therefore

$$
\left|\alpha_{e}-\alpha_{e}^{\prime}\right| \lesssim h^{2+\alpha}, \quad\left|\beta_{e}-\beta_{e}^{\prime}\right| \lesssim h^{2+\alpha} \text {. }
$$

Combining this with (2.6), we have

$$
\left|I_{11}\right| \lesssim h^{1+\alpha}|u|_{2, \infty, \Omega} \int_{\Omega}\left|\nabla v_{h}\right| \lesssim h^{1+\alpha}|u|_{2, \infty, \Omega}\left|v_{h}\right|_{1, \Omega} .
$$


Now we turn to the estimate for $I_{12}$. Since adjacent elements in $\Omega_{2, h}$ do not form an $O\left(h^{1+\alpha}\right)$ approximate parallelogram, we simply estimate

$$
\left|\alpha_{e}-\alpha_{e}^{\prime}\right| \leq\left|\alpha_{e}\right|+\left|\alpha_{e}^{\prime}\right| \lesssim h^{2}, \quad\left|\beta_{e}-\beta_{e}^{\prime}\right| \leq\left|\beta_{e}\right|+\left|\beta_{e}^{\prime}\right| \lesssim h^{2}
$$

Similarly to (2.7), this leads to

$$
\left|I_{12}\right| \lesssim h|u|_{2, \infty, \Omega} \sum_{\tau \in \mathcal{T}_{2, h}} \int_{\tau}\left|\nabla v_{h}\right| \lesssim h|u|_{2, \infty, \Omega}\left\|\nabla v_{h}\right\|_{0, \Omega_{2, h}} h^{\sigma / 2}
$$

Combining this with (2.5) and (2.7) leads to

$$
\left|I_{1}\right| \lesssim h^{1+\rho}|u|_{2, \infty, \Omega}\left|v_{h}\right|_{1, \Omega} .
$$

Finally, applying (2.4) and (2.8) to (2.3), we obtain (2.2).

\section{Gradient ReCOVERY OPERATORS}

We define $\mathcal{N}_{h}$ as the nodal set of a quasi-uniform triangulation $\mathcal{T}_{h}$. Given $z \in \mathcal{N}_{h}$, we consider an element patch $\omega$ around $z$, which we choose as the origin of a local coordinates. Let $\left(x_{j}, y_{j}\right)$ be the barycenter of a triangle $\tau_{j} \subset \omega, j=1,2, \ldots, m$. We require that one of the following two geometric conditions be satisfied for $\alpha \geq 0$ :

$$
\begin{aligned}
& \frac{1}{m} \sum_{j=1}^{m}\left(x_{j}, y_{j}\right)=O\left(h^{1+\alpha}\right)(1,1) . \\
& \sum_{j=1}^{m} \frac{\left|\tau_{j}\right|}{|\omega|}\left(x_{j}, y_{j}\right)=O\left(h^{1+\alpha}\right)(1,1) .
\end{aligned}
$$

Here we use $\left(x_{j}, y_{j}\right)$ to represent a vector in conditions (3.1) and (3.2).

Remark. Condition $(\alpha, \sigma)$ implies both conditions (3.1) and (3.2) for $z \in \mathcal{N}_{h} \cap \Omega_{1, h}$. Indeed, conditions (3.1) and (3.2) are trivially (with $\alpha=\infty$ ) satisfied by uniform meshes of the regular pattern, the Union Jack pattern, and the criss-cross pattern, and allow an $O\left(h^{1+\alpha}\right)$ deviation from those meshes. For example, a strongly regular mesh is an $O\left(h^{2}\right)$ deviation from a uniform mesh of the regular pattern. Note that the condition (3.1) depends only on relative positions of the barycenters of the triangles and is independent of the shapes, sizes, and numbers of those triangles.

A boundary node $z$ usually leads to $\alpha=0$. However, if $z$ is an interior node with $\alpha=0$, then there are no restrictions and we have a completely unstructured mesh around $z$.

Let $u_{I} \in \mathcal{V}_{h}$ be the linear interpolation of a given function $u$. We shall discuss a gradient recovery operator $G_{h}$ and prove the superconvergent property between $\nabla u$ and $G_{h} u_{I}$.

The value of $G_{h} u_{I}$ is first determined at a vertex and then linearly interpolated over the whole domain. There are three popular ways to generate $G_{h} u_{I}$ at a vertex $z$.

(a) Weighted averaging.

$$
G_{h} u_{I}(z)=\sum_{j=1}^{m} \frac{\left|\tau_{j}\right|}{|\omega|} \nabla u_{I}\left(x_{j}, y_{j}\right) .
$$


(b) Local $L^{2}$-projection. We seek linear functions $p_{l} \in P_{1}(\omega)(l=1,2)$, such that

$$
\int_{\omega}\left[p_{l}(x, y)-\partial_{l} u_{I}(x, y)\right] q(x, y) d x d y=0, \quad \forall q \in P_{1}(\omega), \quad l=1,2 .
$$

Then we define $G_{h} u_{I}(z)=\left(p_{1}(0,0), p_{2}(0,0)\right)$.

(c) Local discrete least-squares fitting proposed by Zienkiewicz-Zhu 22]. We seek linear functions $p_{l} \in P_{1}(\omega)(l=1,2)$, such that

$$
\sum_{j=1}^{m}\left[p_{l}\left(x_{j}, y_{j}\right)-\partial_{l} u_{I}\left(x_{j}, y_{j}\right)\right] q\left(x_{j}, y_{j}\right)=0, \quad \forall q \in P_{1}(\omega), \quad l=1,2 .
$$

Then we define $G_{h} u_{I}(z)=\left(p_{1}(0,0), p_{2}(0,0)\right)$.

Note that (c) is a discrete version of (b). The existence and uniqueness of the minimizers in (b) and (c) can be found in [11, Lemma 1]. The following theorem generalizes the result in [11] from $\alpha=1$ to $\alpha>0$.

Theorem 3.1. Let $\omega$ be an element patch around a node $z \in \mathcal{N}_{h}$, let $u \in W_{\infty}^{3}(\omega)$, and let $G_{h} u_{I}(z)$ be produced by either the local $L^{2}$-projection or the weighted averaging under condition (3.2), or by the local discrete least-squares fitting under condition (3.1). Then

$$
\left|G_{h} u_{I}(z)-\nabla u(z)\right| \lesssim h^{1+\alpha}\|u\|_{3, \infty, \omega}
$$

Proof. (a) For the weighted averaging, we have

$$
\begin{aligned}
& \sum_{j=1}^{m} \frac{\left|\tau_{j}\right|}{|\omega|} \partial_{l} u_{I}\left(x_{j}, y_{j}\right)-\partial_{l} u(0,0) \\
= & \left.\sum_{j=1}^{m} \frac{\left|\tau_{j}\right|}{|\omega|} \partial_{l}\left(u_{I}-u\right)\left(x_{j}, y_{j}\right)+\sum_{j=1}^{m} \frac{\left|\tau_{j}\right|}{|\omega|} \partial_{l} u\left(x_{j}, y_{j}\right)-\partial_{l} u(0,0)\right] \\
= & \sum_{j=1}^{m} \frac{\left|\tau_{j}\right|}{|\omega|} \partial_{l}\left(u_{I}-u\right)\left(x_{j}, y_{j}\right)+\nabla \partial_{l} u(0,0) \cdot \sum_{j=1}^{m} \frac{\left|\tau_{j}\right|}{|\omega|}\left(x_{j}, y_{j}\right)+R_{1}(u),
\end{aligned}
$$

where, by the Taylor expansion,

$$
\left|R_{1}(u)\right| \lesssim h^{2}|u|_{3, \infty, \omega}
$$

Since the barycenter is the derivative superconvergent point for the linear interpolation, then

$$
\left|\partial_{l}\left(u_{I}-u\right)\left(x_{j}, y_{j}\right)\right| \lesssim h^{2}|u|_{3, \infty, \omega}, \quad j=1,2, \ldots, m .
$$

Recall the condition (3.2), and we derive

$$
\left|\nabla \partial_{l} u(0,0) \cdot \sum_{j=1}^{m} \frac{\left|\tau_{j}\right|}{|\omega|}\left(x_{j}, y_{j}\right)\right| \lesssim h^{1+\alpha}|u|_{2, \infty, \omega}
$$

Therefore,

$$
\left|\sum_{j=1}^{m} \frac{\left|\tau_{j}\right|}{|\omega|} \partial_{l} u_{I}\left(x_{j}, y_{j}\right)-\partial_{l} u(0,0)\right| \lesssim h^{1+\alpha}\|u\|_{3, \infty, \omega}
$$


(b) For the local $L^{2}$-projection, we set $q=1$ in (3.4) to obtain

$$
\sum_{j=1}^{m}\left|\tau_{j}\right| p_{l}\left(x_{j}, y_{j}\right)=\sum_{j=1}^{m}\left|\tau_{j}\right| \partial_{l} u_{I}\left(x_{j}, y_{j}\right) .
$$

Therefore,

$$
\begin{aligned}
p_{l}(0,0)-\sum_{j=1}^{m} \frac{\left|\tau_{j}\right|}{|\omega|} \partial_{l} u_{I}\left(x_{j}, y_{j}\right) & =p_{l}(0,0)-\sum_{j=1}^{m} \frac{\left|\tau_{j}\right|}{|\omega|} p_{l}\left(x_{j}, y_{j}\right) \\
& =-\nabla p_{l}(0,0) \cdot \sum_{j=1}^{m} \frac{\left|\tau_{j}\right|}{|\omega|}\left(x_{j}, y_{j}\right) .
\end{aligned}
$$

Using (see [11, Lemma 2])

$$
\left|\nabla p_{l}(0,0)\right| \lesssim\|u\|_{3, \infty, \omega}
$$

and condition (3.2), we obtain

$$
\left|p_{l}(0,0)-\sum_{j=1}^{m} \frac{\left|\tau_{j}\right|}{|\omega|} \partial_{l} u_{I}\left(x_{j}, y_{j}\right)\right| \lesssim h^{1+\alpha}\|u\|_{3, \infty, \omega}
$$

Combining (3.6) and (3.8), we have proved

$$
\left|p_{l}(0,0)-\partial_{l} u(0,0)\right| \lesssim h^{1+\alpha}\|u\|_{3, \infty, \omega} .
$$

(c) For the local discrete least-squares fitting, we set $q=1$ in (3.5) to obtain

$$
\sum_{j=1}^{m} p_{l}\left(x_{j}, y_{j}\right)=\sum_{j=1}^{m} \partial_{l} u_{I}\left(x_{j}, y_{j}\right) .
$$

Therefore,

$$
\begin{aligned}
p_{l}(0,0)-\frac{1}{m} \sum_{j=1}^{m} \partial_{l} u_{I}\left(x_{j}, y_{j}\right) & =p_{l}(0,0)-\frac{1}{m} \sum_{j=1}^{m} p_{l}\left(x_{j}, y_{j}\right) \\
& =-\frac{1}{m} \nabla p_{l}(0,0) \cdot \sum_{j=1}^{m}\left(x_{j}, y_{j}\right) .
\end{aligned}
$$

Using (3.7) and condition (3.1), we obtain

$$
\left|p_{l}(0,0)-\frac{1}{m} \sum_{j=1}^{m} \partial_{l} u_{I}\left(x_{j}, y_{j}\right)\right| \lesssim h^{1+\alpha}\|u\|_{3, \infty, \omega} .
$$

Next,

$$
\begin{aligned}
& \frac{1}{m} \sum_{j=1}^{m} \partial_{l} u_{I}\left(x_{j}, y_{j}\right)-\partial_{l} u(0,0) \\
= & \frac{1}{m} \sum_{j=1}^{m} \partial_{l}\left(u_{I}-u\right)\left(x_{j}, y_{j}\right)+\frac{1}{m} \sum_{j=1}^{m}\left[\partial_{l} u\left(x_{j}, y_{j}\right)-\partial_{l} u(0,0)\right] \\
= & \frac{1}{m} \sum_{j=1}^{m} \partial_{l}\left(u_{I}-u\right)\left(x_{j}, y_{j}\right)+\frac{1}{m} \nabla \partial_{l} u(0,0) \cdot \sum_{j=1}^{m}\left(x_{j}, y_{j}\right)+R_{2}(u),
\end{aligned}
$$


with $\left|R_{2}(u)\right| \lesssim h^{2}|u|_{3, \infty, \omega}$. Therefore,

$$
\left|\frac{1}{m} \sum_{j=1}^{m} \partial_{l} u_{I}\left(x_{j}, y_{j}\right)-\partial_{l} u(0,0)\right| \lesssim h^{1+\alpha}\|u\|_{3, \infty, \omega} .
$$

Combining (3.10) and (3.11), we obtain (3.9) for the current case.

Theorem 3.2. The recovery operator $G_{h}$ satisfies

$$
G_{h} v(z)=\sum_{j=1}^{m} c_{j} \nabla v\left(x_{j}, y_{j}\right), \quad \sum_{j=1}^{m} c_{j}=1,
$$

in all three cases unconditionally. Furthermore, $c_{j}>0$ for

(a) the weighted averaging unconditionally;

(b) the local $L^{2}$-projection under the condition (3.2);

(c) the local discrete least-squares fitting under the condition (3.1).

Proof. The assertion is obvious for the weighted averaging case.

Choose $v=x+y$. Then the minimizer $p_{1}=1$ and $p_{2}=1$ in both cases (b) and (c). Therefore,

$$
G_{h} v(z)=(1,1)=\sum_{j=1}^{m} c_{j} \nabla(x+y)=\sum_{j=1}^{m} c_{j}(1,1) .
$$

Now we let $p_{l}(x, y)=a_{0}+a_{1} x+a_{2} y$. Then for the local discrete least-squares fitting, $a_{i}$ 's are given by

$$
\left(\begin{array}{ccc}
m & \sum_{j} x_{j} & \sum_{j} y_{j} \\
\sum_{j} x_{j} & \sum_{j} x_{j}^{2} & \sum_{j} x_{j} y_{j} \\
\sum_{j} y_{j} & \sum_{j} x_{j} y_{j} & \sum_{j} y_{j}^{2}
\end{array}\right)\left(\begin{array}{c}
a_{0} \\
a_{1} \\
a_{2}
\end{array}\right)=\left(\begin{array}{c}
\sum_{j} \partial_{l} u_{h}\left(x_{j}, y_{j}\right) \\
\sum_{j} x_{j} \partial_{l} u_{h}\left(x_{j}, y_{j}\right) \\
\sum_{j} y_{j} \partial_{l} u_{h}\left(x_{j}, y_{j}\right)
\end{array}\right) .
$$

Note that

$$
\sum_{j} x_{j}^{2}=O\left(h^{2}\right), \quad \sum_{j} x_{j} y_{j}=O\left(h^{2}\right), \quad \sum_{j} y_{j}^{2}=O\left(h^{2}\right) ;
$$

and under condition (3.1),

$$
\sum_{j} x_{j}=O\left(h^{1+\alpha}\right), \quad \sum_{j} y_{j}=O\left(h^{1+\alpha}\right) .
$$

By scaling argument we see that

$$
a_{1}=O\left(h^{\alpha-1}\right), \quad a_{2}=O\left(h^{\alpha-1}\right) .
$$

Therefore,

$$
\begin{aligned}
a_{0} & =\frac{1}{m} \sum_{j} \partial_{l} u_{h}\left(x_{j}, y_{j}\right)-\frac{a_{1}}{m} \sum_{j} x_{j}-\frac{a_{2}}{m} \sum_{j} y_{j} \\
& =\sum_{j} c_{j} \partial_{l} u_{h}\left(x_{j}, y_{j}\right)
\end{aligned}
$$

with

$$
c_{j}=\frac{1}{m}+O\left(h^{2 \alpha}\right)>0 .
$$

A similar argument shows that

$$
c_{j}=\frac{\left|\tau_{j}\right|}{|\omega|}+O\left(h^{2 \alpha}\right)>0
$$


for the local $L^{2}$-projection when condition (3.2) is satisfied.

Under the given condition, the recovered gradient at a vertex $z$ is a convex combination of gradient values on the element patch surrounding $z$.

\section{SuperCONVERGENCE OF THE RECOVERY OPERATORS}

We consider the non-self-adjoint problem: find $u \in H^{1}(\Omega)$ such that

$$
B(u, v)=\int_{\Omega}[(\mathcal{D} \nabla u+\boldsymbol{b} u) \cdot \nabla v+c u v]=f(v), \quad \forall v \in H^{1}(\Omega) .
$$

Here $\mathcal{D}$ is a $2 \times 2$ symmetric, positive definite matrix, and $f(\cdot)$ is a linear functional. We assume that all the coefficient functions are smooth, and the bilinear form $B(\cdot, \cdot)$ is continuous and satisfies the inf-sup condition on $H^{1}(\Omega)$. These conditions insure that (4.1) has a unique solution.

The finite element solution $u_{h} \in \mathcal{V}_{h}$ satisfies

$$
B\left(u_{h}, v_{h}\right)=f\left(v_{h}\right) \quad \forall v_{h} \in \mathcal{V}_{h}
$$

To insure a unique solution for (4.2), we further assume the inf-sup condition of $B$ to be satisfied on $\mathcal{V}_{h}$.

We define the piecewise constant matrix function $\mathcal{D}_{\tau}$ in terms of the diffusion matrix $\mathcal{D}$ as follows:

$$
\mathcal{D}_{\tau i j}=\frac{1}{|\tau|} \int_{\tau} \mathcal{D}_{i j} d x
$$

Note that $\mathcal{D}_{\tau}$ is symmetric and positive definite.

Theorem 4.1. Let the solution of (4.1) satisfy $u \in H^{3}(\Omega) \cap W_{\infty}^{2}(\Omega)$, let $u_{h}$ be the solution of (4.2) and let $u_{I} \in \mathcal{V}_{h}$ be the linear interpolation of $u$. Assume that the triangulation $\mathcal{T}_{h}$ satisfies Condition $(\alpha, \sigma)$. Then

$$
\left\|u_{h}-u_{I}\right\|_{1, \Omega} \lesssim h^{1+\rho}\left(\|u\|_{3, \Omega}+|u|_{2, \infty, \Omega}\right), \quad \rho=\min \left(\alpha, \frac{1}{2}, \frac{\sigma}{2}\right) .
$$

Proof. We begin with the identity

$$
\begin{array}{r}
B\left(u-u_{I}, v_{h}\right)=\sum_{\tau \in \mathcal{T}_{h}} \int_{\tau} \nabla\left(u-u_{I}\right) \cdot \mathcal{D}_{\tau} \nabla v_{h} d x+\sum_{\tau \in \mathcal{T}_{h}} \int_{\tau} \nabla\left(u-u_{I}\right) \cdot\left(\mathcal{D}-\mathcal{D}_{\tau}\right) \nabla v_{h} d x \\
+\int_{\Omega}\left(u-u_{I}\right)\left(\boldsymbol{b} \cdot \nabla v_{h}+c v\right) d x=I_{1}+I_{2}+I_{3} .
\end{array}
$$

The first term $I_{1}$ is estimated using Lemma 2.1 and $I_{2}$ and $I_{3}$ can be easily estimated by

$$
\left|I_{2}\right|+\left|I_{3}\right| \lesssim h^{2}\|u\|_{2, \Omega}\|v\|_{1, \Omega}
$$

Thus

$$
\left|B\left(u-u_{I}, v_{h}\right)\right| \lesssim h^{1+\rho}\left(\|u\|_{3, \Omega}+|u|_{2, \infty, \Omega}\right)\left\|v_{h}\right\|_{1, \Omega} .
$$

We complete the proof using the inf-sup condition in

$$
\begin{aligned}
\left\|u_{h}-u_{I}\right\|_{1, \Omega} & \lesssim \sup _{v_{h} \in \mathcal{V}_{h}} \frac{B\left(u_{h}-u_{I}, v_{h}\right)}{\left\|v_{h}\right\|_{1, \Omega}}=\sup _{v_{h} \in \mathcal{V}_{h}} \frac{B\left(u-u_{I}, v_{h}\right)}{\left\|v_{h}\right\|_{1, \Omega}} \\
& \lesssim h^{1+\rho}\left(\|u\|_{3, \Omega}+|u|_{2, \infty, \Omega}\right) .
\end{aligned}
$$


Theorem 4.2. Let the solution of (4.1) satisfy $u \in W_{\infty}^{3}(\Omega)$, let $u_{h}$ be the solution of (4.2), and let $G_{h}$ be a recovery operator defined by one of the three: (a) the weighted averaging, (b) the local $L^{2}$-projection, and (c) the local discrete least-squares fitting. Assume that the triangulation $\mathcal{T}_{h}$ satisfies Condition $(\alpha, \sigma)$. Then

$$
\left\|\nabla u-G_{h} u_{h}\right\|_{0, \Omega} \lesssim h^{1+\rho}\|u\|_{3, \infty, \Omega} .
$$

Proof. We decompose

$$
\nabla u-G_{h} u_{h}=\left(\nabla u-(\nabla u)_{I}\right)+\left((\nabla u)_{I}-G_{h} u_{I}\right)+G_{h}\left(u_{I}-u_{h}\right),
$$

where $(\nabla u)_{I} \in \mathcal{V}_{h} \times \mathcal{V}_{h}$ is the linear interpolation of $\nabla u$. By the standard approximation theory,

$$
\left\|\nabla u-(\nabla u)_{I}\right\|_{0, \Omega} \lesssim h^{2}|u|_{3, \Omega} .
$$

We observe that when we pick an element patch on $\mathcal{T}_{1, h}$, both conditions (3.1) and (3.2) are satisfied. Therefore, using Theorem 3.1, we have

$$
\begin{aligned}
\left\|(\nabla u)_{I}-G_{h} u_{I}\right\|_{0, \Omega_{1, h}} & \leq\left(\sum_{\tau \in \Omega_{1, h}}|\tau| \sum_{z \in \mathcal{N}_{h} \cap \bar{\tau}}\left|G_{h} u_{I}(z)-\nabla u(z)\right|^{2}\right)^{1 / 2} \\
& \lesssim h^{1+\alpha}\|u\|_{3, \infty, \Omega}\left|\Omega_{1, h}\right|^{1 / 2} \lesssim h^{1+\alpha}\|u\|_{3, \infty, \Omega} .
\end{aligned}
$$

On the other hand,

$$
\left\|(\nabla u)_{I}-G_{h} u_{I}\right\|_{0, \Omega_{2, h}} \lesssim h\|u\|_{3, \infty, \Omega}\left|\Omega_{2, h}\right|^{1 / 2} \lesssim h^{1+\sigma / 2}\|u\|_{3, \infty, \Omega}
$$

by Condition $(\alpha, \sigma)$. Combining (4.5) with (4.6), we have

$$
\left\|(\nabla u)_{I}-G_{h} u_{I}\right\|_{0, \Omega} \lesssim h^{1+\min (\alpha, \sigma / 2)}\|u\|_{3, \infty, \Omega} .
$$

Similarly as in (4.5), we have, by using the fact proved in Theorem 3.2, that $G_{h} v(z)$ is a convex combination of $\left.\nabla v\right|_{\tau_{z}}$ 's;

$$
\begin{aligned}
\left\|G_{h}\left(u_{I}-u_{h}\right)\right\|_{0, \Omega_{1, h}} & \leq\left(\sum_{\tau \in \mathcal{T}_{1, h}}|\tau| \sum_{z \in \mathcal{N}_{h} \cap \bar{\tau}}\left|G_{h}\left(u_{I}-u_{h}\right)(z)\right|^{2}\right)^{1 / 2} \\
& \lesssim\left(\left.\sum_{\tau \in \mathcal{T}_{1, h}}|\tau|\left|\nabla\left(u_{I}-u_{h}\right)\right|_{\tau}\right|^{2}\right)^{1 / 2}=\left\|\nabla\left(u_{I}-u_{h}\right)\right\|_{0, \Omega_{1, h}} \\
& \lesssim h^{1+\rho}\|u\|_{3, \infty, \Omega},
\end{aligned}
$$

by Theorem 4.1. In addition,

$$
\begin{aligned}
\left\|G_{h}\left(u_{I}-u_{h}\right)\right\|_{0, \Omega_{2, h}} & \leq\left(\sum_{\tau \in \mathcal{T}_{2, h}}|\tau| \sum_{z \in \mathcal{N}_{h} \cap \bar{\tau}}\left|G_{h}\left(u_{I}-u_{h}\right)(z)\right|^{2}\right)^{1 / 2} \\
& \lesssim h\|u\|_{3, \infty, \Omega}\left(\sum_{\tau \in \mathcal{T}_{2, h}}|\tau|\right)^{1 / 2} \\
& \lesssim h^{1+\sigma / 2}\|u\|_{3, \infty, \Omega}
\end{aligned}
$$

Combining (4.8) and (4.9) yields

$$
\left\|G_{h}\left(u_{I}-u_{h}\right)\right\|_{0, \Omega} \lesssim h^{1+\rho}\|u\|_{3, \infty, \Omega} .
$$


The conclusion follows by applying (4.4), (4.7), and (4.10) to the right-hand side of (4.3).

Theorem 4.2 requires the global regularity $u \in W_{\infty}^{3}(\Omega)$ which is too restrictive in practice. The next theorem turns to interior maximum norm estimates and relaxes the global regularity assumption on the solution.

Theorem 4.3. Consider an interior patch $\omega_{z} \subset \subset \Omega_{d} \subset \Omega_{1, h}$ with $d=\operatorname{dist}\left(\omega_{z}, \partial \Omega_{d}\right)$ $\geq K h$ for some constant $K>0$. Let $u \in W_{\infty}^{2}(\Omega) \cap W_{\infty}^{3}\left(\Omega_{d}\right)$ be the solution of (4.1), let $u_{h}$ be the solution of (4.2), and let $G_{h}$ be a recovery operator defined by one of the three: (a) the weighted averaging, (b) the local $L^{2}$-projection, and (c) the local discrete least-squares fitting. Then we have

$\left|\left(\nabla u-G_{h} u_{h}\right)(z)\right| \lesssim h^{1+\min (1, \alpha)}\|u\|_{3, \infty, \omega_{z}}+d^{-1} h^{2} \ln \frac{1}{h}\|u\|_{2, \infty, \Omega}+h^{1+\alpha} \ln \frac{d}{h}|u|_{2, \infty, \Omega_{d}}$.

Proof. We denote $\mathcal{V}_{h}^{0}\left(\Omega_{d}\right)$ as the finite element subspace that has a compact support on $\Omega_{d}$ and start from

$$
B\left(u_{h}-u_{I}, \chi\right)=B\left(u-u_{I}, \chi\right)=F(\chi), \quad \forall \chi \in \mathcal{V}_{h}^{0}\left(\Omega_{d}\right),
$$

with

$$
F(\chi)=\sum_{e \in \mathcal{E}_{d}} \int_{e} q_{e}\left\{\left(\alpha_{e}-\alpha_{e}^{\prime}\right) \frac{\partial^{2} u}{\partial \boldsymbol{t}^{2}}+\left(\beta_{e}-\beta_{e}^{\prime}\right) \frac{\partial^{2} u}{\partial \boldsymbol{t} \partial \boldsymbol{n}}\right\} \frac{\partial \chi}{\partial \boldsymbol{t}},
$$

where $\mathcal{E}_{d}$ is the edge set of $\Omega_{d}$. By the same argument as in (2.7), we have

$$
|F(\chi)| \lesssim h^{1+\alpha}|u|_{2, \infty, \Omega_{d}} \int_{\Omega_{d}}|\nabla \chi|
$$

Therefore,

$$
|||F|||_{-1, \infty, \Omega_{d}}=\sup _{\chi \in W_{1}^{1}\left(\Omega_{d}\right),|\chi|_{W_{1}^{1}\left(\Omega_{d}\right)}=1} F(\chi) \lesssim h^{1+\alpha}|u|_{2, \infty, \Omega_{d}} .
$$

Recall Theorem 1.2 of Schatz-Wahlbin [13] (it is straightforward to verify that all conditions of that theorem are satisfied under the current situation):

$$
\begin{aligned}
|e|_{W_{\infty}^{1}\left(\Omega_{0}\right)}+d^{-1}\|e\|_{L_{\infty}\left(\Omega_{0}\right)} & \leq C \min _{\chi \in S^{h}}\left(|w-\chi|_{W_{\infty}^{1}\left(\Omega_{d}\right)}+d^{-1}\|w-\chi\|_{L_{\infty}\left(\Omega_{d}\right)}\right) \\
& +C d^{-1-s-N / q}\|e\|_{W_{q}^{-s}\left(\Omega_{d}\right)}+C \ln \frac{d}{h}\left\|F|\||_{-1, \infty, \Omega_{d}},\right.
\end{aligned}
$$

where $e=w-w_{h}$ satisfies $B(e, \chi)=F(\chi)$. Now, setting $q=\infty, s=0, w=0$, $\Omega_{0}=\omega_{z}$, and $w_{h}=u_{I}-u_{h}$, we obtain

$$
\left|u_{h}-u_{I}\right|_{1, \infty, \omega_{z}} \lesssim d^{-1}\left\|u_{h}-u_{I}\right\|_{L_{\infty}\left(\Omega_{d}\right)}+\ln \frac{d}{h}|||F|||_{-1, \infty, \Omega_{d}} .
$$

Applying (4.11) and $\left\|u_{h}-u_{I}\right\|_{L_{\infty}\left(\Omega_{d}\right)} \lesssim h^{2} \ln \frac{1}{h}|u|_{2, \infty, \Omega}$ results in

$$
\left|u_{h}-u_{I}\right|_{1, \infty, \omega_{z}} \lesssim d^{-1} h^{2} \ln \frac{1}{h}\|u\|_{2, \infty, \Omega}+h^{1+\alpha} \ln \frac{d}{h}|u|_{2, \infty, \Omega_{d}} .
$$

Now we decompose

$$
\left(\nabla u-G_{h} u_{h}\right)(z)=\left(\nabla u-G_{h} u_{I}\right)(z)+G_{h}\left(u_{I}-u_{h}\right)(z) .
$$

By Theorem 3.2, $G_{h} v(z)$ is a convex combination of values of $\nabla v$ on $\tau \in \omega_{z}$. Consequently, $G_{h}$ is a bounded operator in the sense

$$
\left|G_{h} v_{h}(z)\right| \lesssim\left|v_{h}\right|_{1, \infty, \omega_{z}}, \quad \forall v_{h} \in \mathcal{V}_{h}
$$


Therefore,

$$
\left|\left(\nabla u-G_{h} u_{h}\right)(z)\right| \lesssim\left|\left(\nabla u-G_{h} u_{I}\right)(z)\right|+\left|u_{I}-u_{h}\right|_{1, \infty, \omega_{z}} .
$$

The conclusion follows by applying Theorem 3.1 and (4.12) to the right-hand side of (4.13).

Remark. When $\alpha<1$, we choose $d=h^{1-\alpha}$ and obtain

$$
\left|\left(\nabla u-G_{h} u_{h}\right)(z)\right| \lesssim h^{1+\alpha} \ln \frac{1}{h} .
$$

When $\alpha \geq 1$, we choose $d=h^{1-\beta}$ with $\beta \in(0,1]$ and obtain

$$
\left|\left(\nabla u-G_{h} u_{h}\right)(z)\right| \lesssim h^{1+\beta} \ln \frac{1}{h} .
$$

We see that when $\alpha \geq 1$, the recovery is more accurate as $z$ leaves the boundary.

\section{AsYmptotic EXACTNESS OF THE RECOVERY TYPE ERROR ESTIMATORS}

With preparation in the previous sections, it is now straightforward to prove the asymptotic exactness of error estimators based on the recovery operator $G_{h}$. The global error estimator is naturally defined by

$$
\eta_{h}=\left\|G_{h} u_{h}-\nabla u_{h}\right\|_{0, \Omega} .
$$

Theorem 5.1. Assume the hypotheses of Theorem 4.2. Furthermore, assume that there exists a constant $c(u)>0$ such that

$$
\left\|\nabla\left(u-u_{h}\right)\right\| \geq c(u) h .
$$

Then

$$
\left|\frac{\eta_{h}}{\left\|\nabla\left(u-u_{h}\right)\right\|_{0, \Omega}}-1\right| \lesssim h^{\rho}, \quad \rho=\min \left(\frac{1}{2}, \frac{\sigma}{2}, \alpha\right) .
$$

Proof. By Theorem 4.2 and hypothesis (5.2), we have

$$
\left|\frac{\eta_{h}}{\left\|\nabla\left(u-u_{h}\right)\right\|_{0, \Omega}}-1\right| \leq \frac{\left\|G_{h} u_{h}-\nabla u\right\|_{0, \Omega}}{\left\|\nabla\left(u-u_{h}\right)\right\|_{0, \Omega}} \lesssim \frac{h^{1+\rho}\|u\|_{3, \infty, \Omega}}{c(u) h} \lesssim h^{\rho} .
$$

The pointwise error estimator at a vertex $z \in \bar{\tau} \subset \Omega_{1, h}$ is naturally defined by

$$
\eta_{h}^{z}=\left|G_{h} u_{h}(z)-\nabla u_{h}(\tau)\right| .
$$

The next theorem shows that the pointwise error estimator is asymptotically exact.

Theorem 5.2. Assume the hypotheses of Theorem 4.3. Let $z$ be a vertex of elements $\tau \subset \Omega_{1, h}$ and assume that there exists a constant $c(u)>0$ such that

$$
\left|\nabla u(z)-\nabla u_{h}(\tau)\right| \geq c(u) h .
$$

Then we have (a) when $\alpha \in(0,1)$,

$$
\left|\frac{\eta_{h}^{z}}{\left|\nabla u(z)-\nabla u_{h}(\tau)\right|}-1\right| \lesssim h^{\alpha},
$$

with $\operatorname{dist}\left(z, \partial \Omega_{1, h}\right) \geq K h^{1-\alpha}$; and (b) when $\alpha \geq 1$,

$$
\left|\frac{\eta_{h}^{z}}{\left|\nabla u(z)-\nabla u_{h}(\tau)\right|}-1\right| \lesssim h^{\beta}, \quad \forall \beta \in(0,1],
$$

with $\operatorname{dist}\left(z, \partial \Omega_{1, h}\right) \geq K h^{1-\beta}$. 
Proof. We only prove the case when $\alpha \in(0,1)$. By Theorem 4.3 and hypothesis (5.4), we have

$$
\left|\frac{\eta_{h}^{z}}{\left|\nabla u(z)-\nabla u_{h}(\tau)\right|}-1\right| \leq \frac{\left|G_{h} u_{h}(z)-\nabla u(z)\right|}{\left|\nabla u(z)-\nabla u_{h}(\tau)\right|} \lesssim \frac{h^{1+\alpha}}{h}=h^{\alpha} .
$$

We see that the error estimators (5.1) and (5.3) based on the gradient recovery operator are asymptotically exact under Condition $(\alpha, \sigma)$. As we mentioned above, this condition is not a very restrictive condition in practice. An automatic mesh generator usually produces some grids which are mildly structured. In practice, a completely unstructured mesh is seldom seen. Our analysis explains in part the good performance of the ZZ error estimator based on the local discrete least-squares fitting for general grids.

\section{ACKNOWLEDGMENTS}

The authors would like to thank Professor Wahlbin for the intriguing discussion which led to the proof of Theorem 4.3.

\section{REFERENCES}

[1] M. Ainsworth and J.T. Oden, A Posteriori Estimation in Finite Element Analysis, Wiley Interscience, New York, 2000. MR 2003b:65001

[2] I. Babuška and W.C. Rheinboldt, A Posteriori Error Estimates for the Finite Element Method, Internat. J. Numer. Methods Engrg., 12 (1978), pp.1597-1615.

[3] I. Babuška and T. Strouboulis, The Finite Element Method and its Reliability, Oxford University Press, London, 2001. MR 2002k:65001

[4] R.E. Bank and A. Weiser, Some a posteriori error estimators for elliptic partial differential equations, Math. Comp., 44 (1985), pp.283-301. MR 86g:65207

[5] R.E. Bank and J. Xu, Asymptotically exact a posteriori error estimators, Part I: Grid with superconvergence, preprint, to appear in SIAM J. Numer. Anal.

[6] R.E. Bank and J. Xu, Asymptotically exact a posteriori error estimators, Part II: General Unstructured Grids, preprint, to appear in SIAM J. Numer. Anal.

[7] C. Carstensen and S. Bartels, Each averaging technique yields reliable a posteriori error control in FEM on unstructured grids. Part I: Low order conforming, nonconforming, and mixed FEM, Math. Comp. 71 (2002), 945-969.

[8] C.M. Chen and Y.Q. Huang, High Accuracy Theory of Finite Element Methods. Hunan Science Press, Hunan, China, 1995 (in Chinese).

[9] W. Hoffmann, A.H. Schatz, L.B. Wahlbin, and G. Wittum, Asymptotically exact a posteriori estimators for the pointwise gradient error on each element in irregular meshes I: A smooth problem and globally quasi-uniform meshes, Math. Comp., 70 (2001), pp.897-909. MR 2002a:65178

[10] M. Kř́žzek, P. Neittaanmäki, and R. Stenberg (Eds.), Finite Element Methods: Superconvergence, Post-processing, and A Posteriori Estimates, Lecture Notes in Pure and Applied Mathematics Series, Vol.196, Marcel Dekker, New York, 1997. MR 98i:65003

[11] B. Li and Z. Zhang, Analysis of a class of superconvergence patch recovery techniques for linear and bilinear finite elements, Numerical Methods for Partial Differential Equations, 15 (1999), pp.151-167. MR 99m:65201

[12] Q. Lin and N. Yan, Construction and Analysis of High Efficient Finite Elements (in Chinese), Hebei University Press, P.R. China, 1996.

[13] A.H. Schatz and L.B. Wahlbin, Interior maximum norm estimates for finite element methods. Part II, Math.Comp., 64 (1995), pp.907-928. MR 95j:65143

[14] R. Verfürth, A Posteriori Error Estimation and Adaptive Mesh Refinement Techniques, Teubner Skripten zur Numerik, B.G. Teubner, Stuttgart, 1995.

[15] L.B. Wahlbin, Superconvergence in Galerkin Finite Element Methods, Lecture Notes in Mathematics, Vol.1605, Springer, Berlin, 1995. MR 98j:65083 
[16] N. Yan and A. Zhou, Gradient recovery type a posteriori error estimates for finite element approximations on irregular meshes, Comput. Methods Appl. Mech. Engrg., 190 (2001), pp.4289-4299. MR 2002c:65189

[17] Z. Zhang, Ultraconvergence of the patch recovery technique II, Math. Comp., 69 (2000), pp.141-158. MR 2000j:65107

[18] Z. Zhang and H.D. Victory Jr., Mathematical Analysis of Zienkiewicz-Zhu's derivative patch recovery techniques, Numerical Methods for Partial Differential Equations, 12 (1996), pp.507524. MR 98c:65191

[19] Q.D. Zhu and Q. Lin, Superconvergence Theory of the Finite Element Method, Hunan Science Press, China, 1989 (in Chinese).

[20] J.Z. Zhu and Z. Zhang, The relationship of some a posteriori error estimators, Comput. Methods Appl. Mech. Engrg., 176 (1999), pp.463-475. MR 2000f:65126

[21] O.C. Zienkiewicz and J.Z. Zhu, A simple error estimator and adaptive procedure for practical engineering analysis, Internat. J. Numer. Methods Engrg., 24 (1987), pp.337-357. MR 87m:73055

[22] O.C. Zienkiewicz and J.Z. Zhu, The superconvergence patch recovery and a posteriori error estimates, Part 1: The recovery technique, Internat. J. Numer. Methods Engrg., 33 (1992), pp.1331-1364. MR 93c:73098

[23] O.C. Zienkiewicz and J.Z. Zhu, The superconvergence patch recovery and a posteriori error estimates. Part 2: Error estimates and adaptivity, Internat. J. Numer. Methods Engrg., 33 (1992), pp.1365-1382. MR 93c:73099

Center for Computational Mathematics and Applications, Department of Mathematics, The Pennsylvania State University, University Park, Pennsylvania 16802

E-mail address: xu@math.psu.edu

Department of Mathematics, Wayne State University, Detroit, Michigan 48202

E-mail address: zzhang@math. wayne.edu 